\title{
Minor Volatile Compounds in White Wines from Canary Islands, Madeira, and Pico (Azores) by Headspace Solid-Phase Microextraction-Gas Chromatography-Mass Spectrometry: A Qualitative Study
}

\author{
S. J. Pérez-Olivero, J. P. Pérez Trujillo, and J. E. Conde \\ Department of Analytical Chemistry, Nutrition and Food Science, University of La Laguna, 38071 La Laguna, Tenerife, Spain
}

Correspondence should be addressed to J. E. Conde; jconde@ull.es

Received 2 September 2013; Accepted 23 September 2013

Academic Editors: P. Campíns-Falcó and P. A. Siskos

Copyright (C) 2013 S. J. Pérez-Olivero et al. This is an open access article distributed under the Creative Commons Attribution License, which permits unrestricted use, distribution, and reproduction in any medium, provided the original work is properly cited.

Application of headspace solid-phase microextraction (HS-SPME) coupled with high-resolution gas chromatographic (HRGC) analytical system was studied for detection and identification of volatile compounds in wines. Four different SPME fibers were tested, and 138 different compounds were detected and identified. The best fiber for the determination of different groups of compounds was selected. Using these results, a comparative study of Madeira, Tenerife (Canary Islands), and Pico (Azores) was carried out.

\section{Introduction}

Wine is an alcoholic solution with a high variety of dissolved substances such as sugars, acids, alcohols, phenolic compounds, nitrogen compounds, macromolecular materials, minerals, and a number of volatile organic compounds that have great influence on sensory and variety characteristics of wine.

Techniques of determinants isolation are a critical step for the determination of aroma compounds. Technique selected can influence the flavor profile obtained; therefore, the analyst should keep in mind every time the advantages and disadvantages of different methods of isolation of these determinants. Selection of sampling techniques, sample preparation, determinants separation, detection, and quantification, will be essential and crucial for the proper chemical characterization of aroma-related compounds.

As aroma compounds are intrinsically volatile substances, most of the compounds should be determined by gas chromatography with the exception for thermolabile substances. In this particular case HPLC or SFC is suitable [1].
SPME technique has a great potential for the analysis of aroma-related compounds. The headspace SPME (HSSPME) avoids the immersion of fiber in complex samples that can reduce fiber life. Besides, gas diffusion coefficients are lower in a gas matrix than in liquid matrix so equilibria are reached earlier for HS-SPME [2]. This is a useful technique for obtaining fingerprints of food flavors, although the most obvious benefit is the ability to isolate and concentrate volatile compounds without interference from matrix components [1]. HS-SPME shows a much higher sensitivity to volatile aromatic compounds and semivolatile than other conventional headspace techniques [3]. In contrast to the extraction techniques based on the total extraction of determinants from the matrix, SPME is based on a balance in the concentrations of the determinants in the sample in the headspace and the fiber stationary phase $[4,5]$. SPME is the suitable technique for volatile compounds in wine. A big number of wine aroma compounds have been characterised by this technique [6-16].

The aim of this work was to apply the GC-MS technique combined with automatic headspace (HS) SPME to the identification of minor volatile compounds in wine selecting the best fiber for this purpose. 


\section{Experimental}

2.1. Chemicals and Reagents. A set of expected wine compounds were purchased from Aldrich (Steinheim, Germany, and Milwaukee, USA), Fluka (Buchs, Switzerland) and Firmenich (Switzerland): $\alpha$-terpineol [7785-53-7], $\beta$-Citronellol [106-22-9], 2,5-dimethyl-4-hydroxy-3-(2H)-furanone [365877-3], 2,6-dimethoxyphenol [91-10-1], 2-ethyl-1-hexanol [10476-7], 2-phenylethanol (phenylethyl alcohol) [60-12-8], 2phenoxyethanol [122-99-6], methyl 4-hydroxybenzoate [9976-3], 4-hydroxy-4-methyl-2-pentanone [123-42-2], butyl acetate [123-86-4], acetoin [513-86-0], isobutyric acid [7931-2], isovaleric acid [503-74-2], succinic acid [110-15-6], benzylic alcohol [100-51-6], benzaldehyde [100-52-7], ethyl benzoate [93-89-0], benzophenone [119-61-9], eugenol [9753-0], guaiacol [90-05-1], ethyl heptanoate [106-30-9], diethyl succinate [123-25-1], E,E-2,4-decadienal [25152-84-5], 4(4-hydroxyphenyl)-2-butanone [5471-51-2], 2-ethyl furoate [614-99-3], 2-methyl-3-furanthiol [28588-74-1], 2-methoxy-4-vinylphenol [7786-61-0], 3-(methylthio)-1-propanol [505-10-2], 4,5-dimethyl-3-hydroxy-2,5-dihydrofuran-2-one [28664-35-9], 4-ethylphenol [123-07-9], 4-ethylguaiacol [2785-89-9], 4-hydroxybenzaldehyde [123-08-0], octanoic acid [124-07-2], butyric acid [107-92-6], decanoic acid [334-48-5], hexanoic acid [142-62-1], acetic acid [64-19-7], propanoic acid [79-09-4], $\alpha$-ionone [127-41-3], $\beta$-ionone [7977-6], Z-3-hexen-1-ol [928-96-1], $\delta$-decalactone [705-86-2], ethyl isovalerate [108-64-5], ethyl vanillate [617-05-0], $\gamma$ decalactone [706-14-9], $\gamma$-hexalactone [695-06-7], $\gamma$-nonalactone [104-61-0], geraniol [106-24-1], isoamyl octanoate [2035-99-6], ethyl isobutyrate [97-62-1], methyl vanillate [3943-74-6], vanillin [121-33-5], vanillin acetone [214-096-9], whiskey lactone [39212-23-2], 2.3-pentanedione [600-14-6], E-2-octenal [2548-87-0], E-2-nonenal [18829-56-6], E,2Z,6-nonadienal [557-48-2], phenylacetaldehyde [122-78-1], 2-aminoacetophenone [613-89-8], 3-aminoacetophenone [99-03-6], 4-aminoacetophenone [99-92-3], hexanal [66-251], E-2-hexen-1-al [6728-26-3], octanal [124-13-0], furfural [98-01-1], decanal [112-31-2], 2-nonanone [821-55-6], 5methylfurfural [620-02-0], acetophenone [98-86-2], ethyl cinnamate [103-36-6], linalool [126-91-0], linalool oxide [60047-17-8], 5-hydroxymethyl-2-furaldehyde [67-47-0], siringaldehyde [134-96-3], 3-methylthio-1-propanol [505-102], 2-methylbutyric acid [116-53-0], theaspirane [36431-72-8], farnesol [4602-84-0], and $\beta$-damascenone [23726-93-4].

Sodium chloride (Merck p.a., Darmstadt, Germany) [7647-14-5], L(+)-tartaric acid [87-69-4] (Merck p.a., Darmstadt, Germany) and sodium hydroxide (Panreac, Barcelona, Spain) [1310-73-2] were used to control ionic strength and to adjust the $\mathrm{pH}$, respectively.

Individual stock standard solutions in ethanol of each compound were prepared. Synthetic matrix solution containing $\mathrm{L}(+)$-tartaric acid $\left(11 \mathrm{~g} \mathrm{~L}^{-1}\right)$ and ethanol (13\%) was prepared for the identification of them in the different wines and adjusted to $\mathrm{pH} 3.2$ with sodium hydroxide. Synthetic and real samples were prepared in $2 \mathrm{~mL}$ vials adding $0.80 \mathrm{~mL}$ of sample and $0.24 \mathrm{~g}$ of $\mathrm{NaCl}$. The vials were tightly capped with PTFE-lined cap and shaken for $10 \mathrm{~min}$ at $200 \mathrm{rpm}$.
2.2. Equipment. Regularly verified pipettes and class A volumetric flasks were used in solution preparation. A precision balance (Sartorius BP 210-S), a pH meter (WTW, pH 197-S), Milli Q-gradient A10 (Millipore), and a mechanical shaker (Selecta, Rotabit) were used in the study.

2.3. SPME Fibers. Four fibers coated with different stationary phases and various film thicknesses were purchased from Supelco (Bellefonte): Polydimethylsiloxane $100 \mu \mathrm{m}$ (PDMS/100), Polydimethylsiloxane-Divinylbenzene $65 \mu \mathrm{m}$ (PDMS/DVB), Polyacrylate $85 \mu \mathrm{m}$ (PA), CarbowaxDivinylbenzene $65 \mu \mathrm{m}$ (CW/DVB). All fibers were conditioned according to manufacturer recommendations.

2.4. Chromatography. The analysis was carried out on a 3800 GC gas chromatograph equipped with an 8200 Standalone autosampler, a 1079 split/splitless injector, and a mass spectrometry detector Saturn 2000 (Varian, Walnut Creek, CA, USA). Injections were performed in splitless mode, using a $0.75 \mathrm{~mm}$ I.D. liner, which improved GC resolution. Ionization mode used was electronic impact.

Separations were performed using a DB-WAXETR capillary column $(60 \mathrm{~m} \times 0.25 \mathrm{~mm}$ I.D., $0.5 \mu \mathrm{m}$ film thickness) (J\&W Scientific) with an injector temperature of $250^{\circ} \mathrm{C}$ (valid for all fibers) and $10 \mathrm{~min}$ of desorption time operating in splitless mode, $60 \mathrm{~min}$ of extraction time, and an oven temperature program of $40^{\circ} \mathrm{C}(15 \mathrm{~min}), 2^{\circ} \mathrm{C} \cdot \mathrm{min}^{-1}, 240^{\circ} \mathrm{C}$, $240^{\circ} \mathrm{C}$ (35 $\left.\mathrm{min}\right)$. Helium was used as carrier at two $\mathrm{mL} \cdot \mathrm{min}^{-1}$ flow.

Peak identification was accomplished using the NIST mass spectra database (Standard Reference Data of National Institute of Standards and Technology, USA) above 95\% of SIM parameter and the synthetic solution of standards.

2.5. Samples. Samples of four different wine varieties, Gual, Malvasía, Listán blanco, and Marmajuelo were studied.

\section{Results and Discussion}

Table 1 shows extracted compounds using different fibers and their retention time. Excluding major compounds, a total of 138 different compounds have been detected, 41 alcohols, 42 esters, 22 aldehydes and ketones, 15 organic acids, 7 hydrocarbons, 9 terpenes, and 3 other compounds.

There is a large number of sensorial important compounds. As expected in young wines, there are many fruity flavor esters. Base aroma compounds were detected including fatty acids and fusel alcohols. These compounds contribute to the balance of fruity sensations. Some detected compounds have been reported having high aroma values or have been of special importance. This group comprises 8 esters (ethyl acetate, phenylethyl acetate, isoamyl acetate, ethyl butyrate, ethyl hexanoate, ethyl octanoate, ethyl isobutyrate, and ethyl isovalerate), two major higher alcohols (isoamyl alcohol and $\beta$-phenylethanol), 8 fatty acids and isoacids 
TABLE 1: Retention time (rt), family classification (F), and detected compounds in the different studied varieties with all fibers. Alcohols (1), esters (2), aldehydes and ketones (3), organic acids (4), hydrocarbons (5), terpenes (6), and other compounds (7).

\begin{tabular}{|c|c|c|c|c|c|c|c|c|c|c|c|c|c|c|c|c|c|c|}
\hline \multirow{2}{*}{ Compounds } & \multirow{2}{*}{$\mathrm{rt}(\min )$} & \multirow{2}{*}{$\mathrm{F}$} & \multicolumn{4}{|c|}{ CW/DVB } & \multicolumn{4}{|c|}{ PDMS/100 } & \multicolumn{4}{|c|}{ PDMS/DVB } & \multicolumn{4}{|c|}{$\mathrm{PA}$} \\
\hline & & & $\mathrm{Gu}$ & $\mathrm{Mv}$ & $\mathrm{Lb}$ & $\mathrm{Mj}$ & $\mathrm{Gu}$ & $\mathrm{Mv}$ & $\mathrm{Lb}$ & $\mathrm{Mj}$ & $\mathrm{Gu}$ & $\mathrm{Mv}$ & $\mathrm{Lb}$ & $\mathrm{Mj}$ & $\mathrm{Gu}$ & $\mathrm{Mv}$ & $\mathrm{Lb}$ & $\mathrm{Mj}$ \\
\hline Isobutyl acetate & 18.68 & 2 & + & + & + & + & + & + & + & + & + & + & + & + & + & + & + & + \\
\hline Ethyl butyrate & 20.83 & 2 & + & + & + & + & + & + & + & + & + & + & + & + & + & + & + & + \\
\hline Ethyl 2-methylbutyrate & 22.23 & 2 & + & + & - & + & + & + & + & + & + & + & + & + & - & + & + & + \\
\hline Ethyl isovalerate & 23.79 & 2 & + & + & + & + & + & + & + & + & + & + & + & + & + & + & + & + \\
\hline Butyl acetate & 24.23 & 2 & + & + & + & + & + & + & + & + & + & + & + & + & + & + & + & + \\
\hline Hexanal & 25.06 & 3 & - & - & - & + & - & - & - & - & + & + & - & - & - & + & - & - \\
\hline 1-Penten-3-ol & 31.89 & 1 & + & + & + & + & + & + & - & - & + & + & + & + & - & + & - & + \\
\hline Ethyl hexanoate & 38.03 & 2 & + & + & + & + & + & + & - & + & + & + & + & + & + & + & + & + \\
\hline 1-Pentanol & 39.13 & 1 & + & + & + & + & + & + & + & + & + & + & + & + & + & + & + & + \\
\hline $\begin{array}{l}\text { Bicyclo } \\
(4,2,0) \text {-Octa-1,3,5-trien }\end{array}$ & 39.91 & 5 & + & + & - & + & + & + & + & + & + & + & + & + & + & + & + & + \\
\hline Hexyl acetate & 41.08 & 2 & + & + & + & + & + & + & + & + & + & + & + & + & + & + & + & + \\
\hline Acetoin & 42.28 & 3 & + & + & + & + & - & + & + & - & + & + & + & + & + & + & + & + \\
\hline 3-Hexen-1-ol acetate & 43.80 & 2 & + & + & + & + & + & + & + & + & + & + & + & + & + & + & + & + \\
\hline Methylcyclopentane & 43.99 & 5 & + & + & + & + & + & + & + & + & + & + & + & + & + & + & + & + \\
\hline 2-Heptanol & 44.47 & 1 & + & + & + & + & + & + & + & + & + & + & + & + & + & + & + & + \\
\hline 4-Methyl-1-pentanol & 44.95 & 1 & + & + & + & + & + & + & + & + & + & + & + & + & + & + & + & + \\
\hline Ethyl heptanoate & 45.55 & 2 & + & + & + & + & + & + & + & + & + & + & + & + & + & + & + & + \\
\hline Ethyl lactate & 46.50 & 2 & + & + & + & + & + & + & + & + & + & + & + & + & + & + & + & + \\
\hline 1-Hexanol & 46.96 & 1 & + & + & + & + & + & + & + & + & + & + & + & + & + & + & + & + \\
\hline 3-Hexen-1-ol & 47.66 & 1 & + & + & + & + & + & + & + & + & + & + & + & + & + & + & + & + \\
\hline 3-Ethoxy-1-propanol & 48.49 & 1 & + & + & + & + & + & + & + & + & + & + & + & + & + & + & + & + \\
\hline Z-3-Hexen-1-ol & 49.29 & 1 & + & + & + & + & + & + & + & + & + & + & + & + & + & + & + & + \\
\hline Methyl heptanoate & 49.59 & 2 & + & + & + & + & + & + & + & + & + & + & + & + & + & + & + & + \\
\hline 3-Octanol & 49.63 & 1 & - & + & + & + & - & + & + & + & + & + & + & + & + & + & + & + \\
\hline 2-Nonen-1-ol & 49.92 & 1 & + & + & + & + & + & + & + & + & + & + & + & + & + & + & + & + \\
\hline 2-Hexen-1-ol & 51.28 & 1 & + & + & + & + & + & - & + & + & + & + & + & + & + & + & + & + \\
\hline Ethyl octanoate & 52.64 & 2 & + & + & + & + & + & + & + & + & + & + & + & + & + & + & + & + \\
\hline E-Linalool oxide & 53.12 & 6 & + & + & + & + & - & + & + & - & + & + & + & + & + & + & + & + \\
\hline 1-Octen-3-ol & 53.60 & 1 & + & + & + & + & + & + & + & + & + & + & + & + & + & + & + & + \\
\hline 1-Heptanol & 53.88 & 1 & + & + & + & + & + & + & + & + & + & + & + & + & + & + & + & + \\
\hline Acetic acid & 53.94 & 4 & + & + & + & + & + & + & + & + & + & + & + & + & + & + & + & + \\
\hline 2-Furaldehyde & 55.02 & 3 & + & + & + & + & + & + & + & + & + & + & + & + & + & + & + & + \\
\hline 3-Hepten-1-ol & 56.51 & 1 & - & + & + & - & - & + & - & + & + & + & + & + & + & + & + & - \\
\hline 2-Decen-1-ol & 56.98 & 1 & + & + & + & + & + & + & + & + & + & + & + & + & + & + & + & + \\
\hline Theaspirane I & 57.19 & 5 & + & + & + & + & + & - & + & + & + & + & + & + & + & + & + & + \\
\hline $\begin{array}{l}\text { (s)-3-Ethyl-4- } \\
\text { methylpentanol }\end{array}$ & 57.45 & 1 & + & + & + & + & + & + & + & + & + & + & + & + & + & + & + & + \\
\hline 2-Nonanol & 58.01 & 1 & + & + & + & + & + & + & + & + & + & + & + & + & + & + & + & + \\
\hline $\begin{array}{l}\text { Ethyl-3- } \\
\text { hydroxybutyrate }\end{array}$ & 58.22 & 2 & + & + & + & + & + & + & + & + & + & + & + & + & + & + & + & + \\
\hline Benzaldehyde & 59.02 & 3 & + & + & + & + & + & + & + & + & + & + & + & + & + & + & + & + \\
\hline Ethyl nonanoate & 59.25 & 2 & + & + & + & + & + & + & + & + & + & + & + & + & + & + & + & + \\
\hline E-2-Nonenal & 59.42 & 3 & + & + & + & + & - & + & + & - & - & + & + & - & - & + & - & + \\
\hline Propionic acid & 59.57 & 4 & + & + & + & + & + & + & + & + & + & + & + & + & + & + & + & + \\
\hline
\end{tabular}


TABLE 1: Continued.

\begin{tabular}{|c|c|c|c|c|c|c|c|c|c|c|c|c|c|c|c|c|c|c|}
\hline \multirow{2}{*}{ Compounds } & \multirow{2}{*}{$\mathrm{rt}(\mathrm{min})$} & \multirow{2}{*}{$\mathrm{F}$} & \multicolumn{4}{|c|}{ CW/DVB } & \multicolumn{4}{|c|}{ PDMS/100 } & \multicolumn{4}{|c|}{ PDMS/DVB } & \multicolumn{4}{|c|}{$\mathrm{PA}$} \\
\hline & & & $\mathrm{Gu}$ & $\mathrm{Mv}$ & $\mathrm{Lb}$ & $\mathrm{Mj}$ & $\mathrm{Gu}$ & $\mathrm{Mv}$ & $\mathrm{Lb}$ & $\mathrm{Mj}$ & $\mathrm{Gu}$ & $\mathrm{Mv}$ & $\mathrm{Lb}$ & $\mathrm{Mj}$ & $\mathrm{Gu}$ & $\mathrm{Mv}$ & $\mathrm{Lb}$ & $\mathrm{Mj}$ \\
\hline Theaspirane II & 59.65 & 5 & + & + & - & + & + & + & + & + & + & + & + & + & + & - & + & + \\
\hline Linalool & 59.90 & 6 & + & + & + & + & + & + & + & + & + & + & + & + & + & + & + & + \\
\hline 1-Octanol & 60.53 & 1 & + & + & + & + & + & + & + & + & + & + & + & + & + & + & + & + \\
\hline Isobutyric acid & 61.42 & 4 & + & + & + & + & + & + & + & + & + & + & + & + & + & + & + & + \\
\hline Diethyl malonate & 62.19 & 2 & + & + & + & + & - & + & + & + & - & + & + & + & + & + & + & + \\
\hline E-2,Z-6-Nonadienal & 62.70 & 3 & + & - & - & - & - & - & - & - & - & - & - & - & - & - & - & - \\
\hline $\begin{array}{l}\text { 2-Methylundecanoic } \\
\text { acid }\end{array}$ & 62.95 & 4 & + & + & + & + & + & + & + & + & + & + & + & + & + & + & + & + \\
\hline $\begin{array}{l}\text { 1,5,7-Octatrien-3-ol- } \\
\text { 3,7-dimethyl }\end{array}$ & 63.87 & 1 & + & + & + & + & + & + & + & + & + & + & + & + & + & + & + & + \\
\hline Cyclooctyl alcohol & 64.11 & 1 & + & + & + & + & - & + & - & - & + & + & + & + & + & + & + & - \\
\hline Ethoxytriglycol & 64.40 & 1 & + & + & + & + & - & + & + & - & + & + & + & + & + & - & + & + \\
\hline Butyric acid & 65.05 & 4 & + & + & + & + & + & + & + & + & + & + & + & + & + & + & + & + \\
\hline Ethyl 2-furoate & 65.06 & 2 & + & + & + & + & + & + & + & + & + & + & + & + & + & + & + & + \\
\hline Methyl benzoate & 65.10 & 2 & - & - & - & - & - & - & - & + & + & + & + & + & + & + & + & + \\
\hline Ethyl decanoate & 65.45 & 2 & + & + & + & + & + & + & + & + & + & + & + & + & + & + & + & + \\
\hline 2-Decenal & 66.01 & 3 & + & + & + & - & - & + & - & - & + & + & + & + & + & + & - & - \\
\hline Phenylacetaldehyde & 66.44 & 3 & + & + & + & + & + & + & + & + & + & + & + & + & + & + & + & + \\
\hline Isoamyl octanoate & 66.59 & 2 & + & + & + & + & + & + & + & + & + & + & + & + & + & + & + & + \\
\hline Acetophenone & 66.82 & 3 & + & + & + & + & + & + & + & + & + & + & + & + & + & + & + & + \\
\hline Isovaleric acid & 67.62 & 4 & + & + & + & + & + & + & + & + & + & + & + & + & + & + & + & + \\
\hline Ethyl benzoate & 67.87 & 2 & + & + & + & + & + & + & + & + & + & + & + & + & + & + & + & + \\
\hline 2-Methylbutyric acid & 67.87 & 4 & + & + & + & + & + & + & + & + & + & + & + & + & + & + & + & + \\
\hline Diethyl succinate & 67.97 & 2 & + & + & + & + & + & + & + & + & + & + & + & + & + & + & + & + \\
\hline 4-Decen-1-ol (Z) & 68.18 & 1 & + & + & + & + & + & + & + & + & + & + & + & + & + & + & + & + \\
\hline Ethyl 4-decenoate & 68.62 & 2 & + & + & + & + & + & + & + & + & + & + & + & + & + & + & + & + \\
\hline$\alpha$-Terpineol & 69.13 & 6 & + & + & + & + & + & + & + & + & + & + & + & + & + & + & + & + \\
\hline$\gamma$-Hexalactone & 69.72 & 3 & + & + & + & + & + & + & + & + & + & + & + & + & + & + & + & + \\
\hline E-2-Decen-1-ol & 69.80 & 1 & + & + & + & + & + & + & + & + & + & + & + & + & + & + & + & + \\
\hline $\begin{array}{l}\text { 3-(Methylthio)-1- } \\
\text { propanol }\end{array}$ & 70.25 & 1 & + & + & + & + & + & + & + & + & + & + & + & + & + & + & + & + \\
\hline Benzyl acetate & 71.27 & 2 & + & + & - & + & + & + & + & + & + & - & + & + & + & + & + & + \\
\hline $\begin{array}{l}\text { 1,3-Propanediol } \\
\text { diacetate }\end{array}$ & 71.40 & 2 & + & + & + & + & + & + & + & + & + & + & + & + & + & + & + & + \\
\hline 1-Decanol & 72.59 & 1 & + & + & + & + & + & + & + & + & + & + & + & + & + & + & + & + \\
\hline$\beta$-Citronellol & 72.87 & 6 & + & + & + & + & + & + & + & + & + & + & + & + & + & + & + & + \\
\hline Methyl salicylate & 73.95 & 2 & + & + & + & + & + & + & + & + & + & + & + & + & + & + & + & + \\
\hline 1-Dodecene & 74.18 & 5 & - & + & - & + & + & + & - & - & + & - & + & + & + & + & + & + \\
\hline Phenylacetic acid & 74.35 & 4 & + & + & + & + & + & + & + & + & + & + & + & + & + & + & + & + \\
\hline Z-Geraniol & 74.77 & 6 & + & + & + & + & + & + & + & + & + & + & + & + & + & + & + & + \\
\hline 5-Mercapto-1-pentanol & 75.02 & 1 & + & + & + & + & + & + & + & + & + & + & + & + & + & + & + & + \\
\hline E,E-2,4-Decadienal & 75.63 & 3 & + & - & - & - & - & - & - & - & - & - & - & + & + & + & - & + \\
\hline 1-Phenylethanol & 75.70 & 1 & - & - & + & - & + & - & + & + & - & - & + & - & + & + & + & + \\
\hline$\beta$-Phenylacetate & 76.08 & 2 & + & + & + & + & + & + & + & + & + & + & + & + & + & + & + & + \\
\hline$\beta$-Damascenone & 76.25 & 3 & + & + & + & + & + & + & + & + & + & + & + & + & + & + & + & + \\
\hline Ethyl dodecanoate & 76.97 & 2 & + & + & + & + & + & + & + & + & + & + & + & + & + & + & + & + \\
\hline
\end{tabular}


TABle 1: Continued.

\begin{tabular}{|c|c|c|c|c|c|c|c|c|c|c|c|c|c|c|c|c|c|c|}
\hline \multirow{2}{*}{ Compounds } & \multirow{2}{*}{$\mathrm{rt}(\min )$} & \multirow{2}{*}{$\mathrm{F}$} & \multicolumn{4}{|c|}{ CW/DVB } & \multicolumn{4}{|c|}{ PDMS/100 } & \multicolumn{4}{|c|}{ PDMS/DVB } & \multicolumn{4}{|c|}{ PA } \\
\hline & & & $\mathrm{Gu}$ & $\mathrm{Mv}$ & $\mathrm{Lb}$ & $\mathrm{Mj}$ & $\mathrm{Gu}$ & $\mathrm{Mv}$ & $\mathrm{Lb}$ & $\mathrm{Mj}$ & $\mathrm{Gu}$ & $\mathrm{Mv}$ & $\mathrm{Lb}$ & $\mathrm{Mj}$ & $\mathrm{Gu}$ & $\mathrm{Mv}$ & $\mathrm{Lb}$ & $\mathrm{Mj}$ \\
\hline Hexanoic acid & 77.25 & 4 & + & + & + & + & + & + & + & + & + & + & + & + & + & + & + & + \\
\hline$\alpha$-Ionone & 77.76 & 6 & + & + & + & + & + & + & + & + & + & + & + & + & + & + & + & + \\
\hline Dihydropseudoionone & 77.85 & 3 & + & + & + & + & + & + & + & + & + & + & + & + & + & + & + & + \\
\hline $\begin{array}{l}\mathrm{N}(3 \text {-methylbutyl) } \\
\text { acetamide }\end{array}$ & 77.89 & 7 & + & + & + & + & + & + & + & - & + & + & + & - & + & + & + & - \\
\hline $\begin{array}{l}\text { 3-Methylbutyl } \\
\text { pentadecanoate }\end{array}$ & 77.94 & 2 & + & + & + & + & + & + & + & + & + & + & + & + & + & + & + & + \\
\hline Guaiacol & 78.55 & 1 & - & + & + & + & - & - & + & - & + & + & + & - & + & + & + & - \\
\hline 4,5-Dimethyl-1-hexene & 78.79 & 5 & + & + & + & + & + & + & + & + & + & + & + & + & + & + & + & + \\
\hline Benzylic alcohol & 79.34 & 1 & + & + & + & + & + & + & + & + & + & + & + & + & + & + & + & + \\
\hline Octyl acetate & 79.38 & 2 & + & + & + & + & + & + & + & + & + & + & + & + & + & + & + & + \\
\hline Ethyl hydrocinnamate & 79.73 & 2 & + & + & + & + & + & + & + & - & - & + & + & + & + & + & + & + \\
\hline Whiskey lactone I & 79.93 & 3 & + & + & - & + & - & - & - & - & + & - & + & + & + & + & - & + \\
\hline 2-Phenylethanol & 81.15 & 1 & - & - & - & - & + & + & + & + & + & + & + & + & + & + & + & + \\
\hline$\gamma$-Octalactone & 81.53 & 3 & + & + & + & + & + & + & + & + & + & + & + & + & + & + & + & + \\
\hline Ethyl palmitate & 82.35 & 2 & + & + & - & + & - & - & - & - & + & + & + & + & + & - & + & + \\
\hline$\beta$-Ionone & 82.38 & 6 & + & - & - & - & + & - & + & + & + & - & + & + & + & - & + & + \\
\hline 2-Ethylhexanoic acid & 82.64 & 4 & + & + & + & + & + & + & + & + & + & + & + & + & + & + & + & + \\
\hline Heptanoic acid & 82.82 & 4 & + & + & + & + & + & + & + & + & + & + & + & + & + & + & + & + \\
\hline o-Cresol & 85.65 & 1 & + & + & + & + & - & - & - & - & + & + & + & - & + & + & + & + \\
\hline Phenol & 85.84 & 1 & + & + & + & + & + & + & + & + & + & + & + & + & + & + & + & + \\
\hline Isopropyl myristate & 86.81 & 2 & + & + & + & + & + & + & + & + & + & + & + & + & + & + & + & + \\
\hline$\gamma$-Nonalactone & 87.05 & 3 & + & + & + & + & + & + & + & + & + & + & + & + & + & + & + & + \\
\hline Furaneol & 87.11 & 3 & + & + & + & + & + & + & + & + & + & - & + & + & + & + & + & + \\
\hline 4-Ethylguaiacol & 87.22 & 1 & - & - & - & - & - & - & - & - & - & - & - & - & - & - & - & - \\
\hline Ethyl myristate & 87.49 & 2 & + & + & + & + & + & + & + & + & + & + & + & + & + & + & + & + \\
\hline Octanoic acid & 88.46 & 4 & + & + & + & + & + & + & + & + & + & + & + & + & + & + & + & + \\
\hline $\begin{array}{l}\text { Isoaromadendrene } \\
\text { epoxide }\end{array}$ & 89.04 & 7 & + & + & - & + & + & - & + & + & + & - & - & + & + & - & - & + \\
\hline m-Cresol & 90.19 & 1 & - & + & - & - & - & - & - & - & - & - & - & - & + & + & + & + \\
\hline Ethyl cinnamate & 92.04 & 2 & + & + & + & + & + & + & + & + & + & + & + & + & + & + & + & + \\
\hline Ethyl pentadecanoate & 92.37 & 2 & - & + & + & + & + & + & + & - & + & + & - & + & + & + & + & + \\
\hline 2-Phenoxyethanol & 92.62 & 1 & - & - & - & - & - & - & - & - & - & - & - & - & - & - & - & - \\
\hline$\gamma$-Decalactone & 92.70 & 3 & + & + & + & + & + & + & + & + & + & + & + & + & + & + & + & + \\
\hline Nonanoic acid & 93.18 & 4 & + & + & + & + & + & + & + & + & + & + & + & + & + & + & + & + \\
\hline $\begin{array}{l}\text { N-Morfolinomethyl- } \\
\text { isopropylsulfur }\end{array}$ & 93.47 & 7 & + & + & + & + & + & + & + & + & + & + & + & + & + & + & + & + \\
\hline Eugenol & 93.77 & 2 & + & + & + & + & + & + & - & + & + & + & - & + & + & + & - & + \\
\hline 4-Ethylphenol & 94.16 & 1 & + & + & - & + & + & - & - & - & + & + & - & - & + & + & + & + \\
\hline$\delta$-Decalactone & 95.02 & 3 & - & + & + & + & + & + & + & + & + & - & - & - & - & - & - & - \\
\hline $\begin{array}{l}\text { 2-Methoxy-4- } \\
\text { vinylphenol }\end{array}$ & 95.16 & 1 & + & + & + & + & + & + & + & + & + & + & + & + & + & + & + & + \\
\hline Ethyl hexadecanoate & 97.06 & 2 & + & + & + & + & + & + & + & + & + & + & + & + & + & + & + & + \\
\hline 1-Hepten-4-ol & 97.43 & 1 & + & + & + & + & - & - & - & - & - & + & - & + & + & + & - & - \\
\hline Decanoic acid & 97.99 & 4 & + & + & + & + & + & + & + & + & + & + & + & + & + & + & + & + \\
\hline$\gamma$-Undecalactone & 97.99 & 3 & + & + & + & + & + & + & + & + & + & + & + & + & + & + & + & + \\
\hline Dimethyl phthalate & 99.62 & 2 & + & + & - & + & + & + & + & + & + & + & + & + & + & + & - & + \\
\hline
\end{tabular}


TABLe 1: Continued.

\begin{tabular}{|c|c|c|c|c|c|c|c|c|c|c|c|c|c|c|c|c|c|c|}
\hline \multirow{2}{*}{ Compounds } & \multirow{2}{*}{$\mathrm{rt}(\mathrm{min})$} & \multirow{2}{*}{$\mathrm{F}$} & \multicolumn{4}{|c|}{ CW/DVB } & \multicolumn{4}{|c|}{ PDMS/100 } & \multicolumn{4}{|c|}{ PDMS/DVB } & \multicolumn{4}{|c|}{ PA } \\
\hline & & & $\mathrm{Gu}$ & $\mathrm{Mv}$ & $\mathrm{Lb}$ & $\mathrm{Mj}$ & $\mathrm{Gu}$ & $\mathrm{Mv}$ & $\mathrm{Lb}$ & $\mathrm{Mj}$ & $\mathrm{Gu}$ & $\mathrm{Mv}$ & $\mathrm{Lb}$ & $\mathrm{Mj}$ & $\mathrm{Gu}$ & $\mathrm{Mv}$ & $\mathrm{Lb}$ & $\mathrm{Mj}$ \\
\hline Farnesol I & 99.92 & 6 & - & - & - & + & - & + & - & - & - & - & - & - & - & + & - & - \\
\hline Farnesol III & 101.66 & 6 & + & + & + & + & + & + & + & + & - & + & + & + & + & + & + & + \\
\hline 1-Octadecene & 102.54 & 5 & + & + & + & + & + & + & + & + & + & + & + & + & + & + & + & + \\
\hline Furasol & 103.08 & 1 & + & + & + & + & + & + & + & + & + & + & + & + & + & + & + & + \\
\hline 4-Vinylphenol & 103.89 & 1 & + & + & + & + & + & + & + & + & + & + & + & + & + & + & + & + \\
\hline Ethyl octadecanoate & 105.90 & 2 & - & - & + & - & - & + & - & - & + & + & + & + & - & + & + & - \\
\hline Benzoic acid & 105.95 & 4 & + & + & + & + & + & + & + & + & + & + & + & + & + & + & + & + \\
\hline Benzophenone & 107.57 & 3 & + & + & + & + & + & + & + & + & - & - & - & - & + & + & + & + \\
\hline 2-Monolinolein & 108.81 & 2 & - & - & - & - & - & + & - & - & - & - & - & - & - & + & - & - \\
\hline $\begin{array}{l}\text { Butyl 2-ethylhexyl } \\
\text { phthalate }\end{array}$ & 109.63 & 2 & + & + & + & + & + & + & + & + & + & + & + & + & + & + & + & + \\
\hline Vanillin & 110.89 & 3 & + & + & - & + & + & + & + & + & + & + & + & - & + & - & - & + \\
\hline Octyl butyl phthalate & 115.77 & 2 & + & + & + & + & + & + & + & + & + & + & + & + & + & + & + & + \\
\hline
\end{tabular}

(propanoic, butyric, hexanoic, octanoic, decanoic, isobutyric, 2-methylbutyric, and isovaleric), 2 volatile phenols (4vinylphenol and 4 -vinylguaiacol) as well as linalool and $\beta$ damascenone $[17,18]$.

The presence of $\beta$-damascenone in all studied wines is remarkable. It is considered a high quality aroma, reminiscent of exotic flowers and fruit. $\beta$-Damascenone appears during must fermentation as a by-product of hydrolysis. However, its presence could be related to the particular climate of the Canary Islands that promotes the synthesis of norisoprenoids in grapes $[19,20]$.

Some fibers show little ability in extracting some compounds. CW/DVB fiber does not extract the following compounds from any of the wines: methyl benzoate, 4-ethylguaiacol, 2-phenoxyethanol, and 2-monolinolein. PDMS/100 fiber does not extract hexanal, E,2-Z,6-nonadienal, whiskey lactone I, o-cresol, m-cresol, ethyl palmitate, 4-ethylguaiacol, 2-phenoxyethanol, or 1-hepten-4-ol. The same happens when using PDMS/DVB fiber and E,2-Z,6-nonadienal, 4-ethylguaiacol, m-cresol, 2-phenoxyethanol, farnesol I, benzophenone, and 2-monolinolein. In relation to the PA fiber does not extract the E,2-Z,6-nonadienal, 4-ethylguaiacol, 2-phenoxyethanol, and $\delta$-decalactone.

Farnesol I and 2-monolinolein were not detected in Gual wine, E,E-2,4-decadienal, farnesol I, and 2-monolinolein were not detected in Listán blanco wine, and E,2-Z,6nonadienal and 2-monolinolein were not detected in Marmajuelo wine. On the other hand, 2-monolinolein was only detected in Malvasía wine.

Variability in number and compounds was detected when different wines and fibers were used. Table 2 shows the number of compounds extracted for each wine variety and fiber.

Sum of absolute peak area and number of detected compounds along the four wines were used to select the best fiber for each chemical family. When overall compounds were taken in account PA shows the best efficiency followed by PDMS/DVB, CW/DVB, and PDMS/100, respectively, either for number of compound or peak areas. This pattern is similar for alcohols with slight differences.

Esters present the same number of extracted compounds for PA and PDMS/DVB. However, PDMS/DVB shows higher peak areas.

Carbonylic (aldehydes + ketones) compounds were best extracted by CW/DVB followed by PA, PDMS/DVB, and PDMS/100, respectively, either accounting sum of extracted compounds or sum of peak areas.

Hydrocarbon compounds were best extracted with PA fiber. This fiber extracts the higher number of compounds and the higher peak areas too. However, the rest of fibers do not show any pattern.

Acids present the same number of compounds extracted with all fibers but PA presents the higher sum of areas.

Terpene compounds do not show any clear pattern either by sum of areas or by sum of extracted compounds.

Finally, the group catalogued as others was best extracted by CW/DVB fiber.

From all these pieces of information, we can conclude that $\mathrm{PA}$ is the more suitable fiber for extracting aroma compounds present in wines. Only carbonylic compounds present better results for CW/DVB than for PA fiber.

Extraction information was analyzed attending to different wines studied. Results are shown in Table 3. PA fiber allows the detection of more compounds in Gual and Malvasía wines, PDMS/DVB fiber in Listán blanco wine, and CW/DVB fiber in Marmajuelo wine. On the other hand, all fibers allow the detection of the highest number of compounds in Malvasía wine. These results indicate that Malvasía wine presents the higher concentrations of compounds among the four wines used for the study. On the other hand, PDMS/100 fiber offers the lowest number of compounds detected in all wines suggesting that it is the worst fiber for this kind of samples.

Once best fibers were selected, PA was used in a comparative study among different wines from Pico (Azores), Madeira, and Tenerife Islands. Three different wine varieties 
TABLE 2: Sumatories of number of detected and identified compounds and total area for studied chemical families.

\begin{tabular}{|c|c|c|}
\hline Fiber & No. of compounds & Total area \\
\hline \multicolumn{3}{|c|}{ Overall compounds } \\
\hline $\mathrm{PA}$ & 482 & $4.12 E+08$ \\
\hline $\mathrm{CW} / \mathrm{DVB}$ & 469 & $3.18 E+08$ \\
\hline PDMS/100 & 444 & $2.62 E+08$ \\
\hline PDMS/DVB & 471 & $3.87 E+08$ \\
\hline \multicolumn{3}{|c|}{ Alcohols } \\
\hline $\mathrm{PA}$ & 133 & $1.25 E+08$ \\
\hline $\mathrm{CW} / \mathrm{DVB}$ & 125 & $1.04 E+08$ \\
\hline PDMS/100 & 111 & $6.58 E+07$ \\
\hline PDMS/DVB & 125 & $3.69 E+07$ \\
\hline \multicolumn{3}{|c|}{ Esters } \\
\hline $\mathrm{PA}$ & 156 & $1.70 E+08$ \\
\hline CW/DVB & 148 & $1.47 E+08$ \\
\hline PDMS/100 & 147 & $1.64 E+08$ \\
\hline PDMS/DVB & 156 & $1.98 E+08$ \\
\hline \multicolumn{3}{|c|}{ Carbonylic } \\
\hline $\mathrm{PA}$ & 65 & $4.43 E+06$ \\
\hline $\mathrm{CW} / \mathrm{DVB}$ & 71 & $4.98 E+06$ \\
\hline PDMS/100 & 61 & $2.23 E+06$ \\
\hline PDMS/DVB & 64 & $4.28 E+06$ \\
\hline \multicolumn{3}{|c|}{ Hydrocarbons } \\
\hline $\mathrm{PA}$ & 27 & $1.20 E+07$ \\
\hline $\mathrm{CW} / \mathrm{DVB}$ & 24 & $8.53 E+06$ \\
\hline PDMS/100 & 25 & $2.30 E+06$ \\
\hline PDMS/DVB & 27 & $5.75 E+06$ \\
\hline \multicolumn{3}{|c|}{ Acids } \\
\hline $\mathrm{PA}$ & 60 & $9.57 E+07$ \\
\hline $\mathrm{CW} / \mathrm{DVB}$ & 60 & $8.50 E+07$ \\
\hline PDMS/100 & 60 & $5.28 E+07$ \\
\hline PDMS/DVB & 60 & $6.95 E+07$ \\
\hline \multicolumn{3}{|c|}{ Terpenes } \\
\hline $\mathrm{PA}$ & 32 & $2.05 E+06$ \\
\hline $\mathrm{CW} / \mathrm{DVB}$ & 30 & $3.31 E+06$ \\
\hline PDMS/100 & 30 & $4.06 E+06$ \\
\hline PDMS/DVB & 30 & $4.39 E+06$ \\
\hline \multicolumn{3}{|c|}{ Others } \\
\hline $\mathrm{PA}$ & 9 & $2.22 E+06$ \\
\hline $\mathrm{CW} / \mathrm{DVB}$ & 11 & $2.37 E+06$ \\
\hline PDMS100 & 10 & $1.96 E+06$ \\
\hline PDMS-DVB & 9 & $1.25 E+06$ \\
\hline
\end{tabular}

were used, Gual, Verdelho, and Malvasía. Verdelho wine is produced in the three islands, but Gual and Malvasía are only present in Tenerife and Madeira.

Figures one to three show the sum of areas of different compounds families for Gual, Malvasía, and Verdelho wines.

Madeira Gual wines showed higher values for all families than Tenerife wines except for alcohols. Higher differences appear in esters and carbonylic compounds (Figure 1).

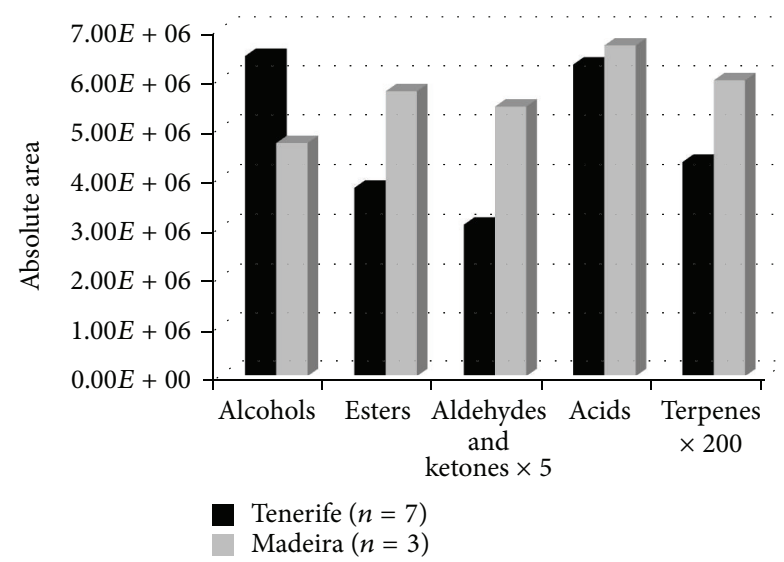

FIgUre 1: Absolute areas for different compound families in Gual wines.

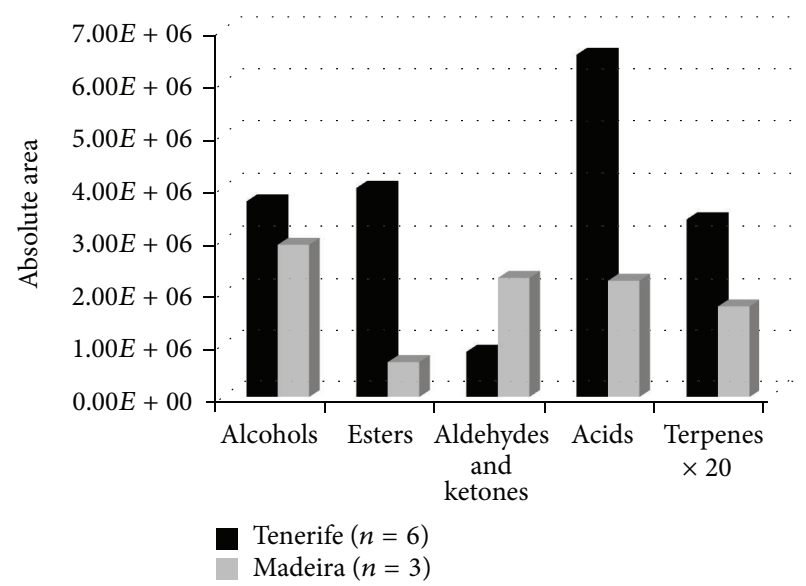

FIgURE 2: Absolute areas for different compound families in Malvasía wines.

Different patter is shown in Figure 2 for Malvasía wines. Tenerife wines showed higher peak areas for all families except for aldehydes and ketones. This is especially remarkable for acids and esters. Highest areas are presented for alcohols in Madeira wines and acids in Tenerife wines. Minimum area is presented for terpenes in both locations (Figure 2).

Figure 3 presents results for Verdelho wines. Wines from Madeira and Tenerife islands present peak areas much higher than wines from Pico Island for acid compounds.

Esters are higher in Tenerife Verdelho wines than in the rest of Islands. Opposite to the other varieties, alcohols for Madeira and Pico wines present higher peak areas than Tenerife wines. Terpenes continue presenting the lower peak areas.

\section{Conclusions}

The SPME coupled to gas chromatography-mass spectrometry detector is a useful technique for identification of minor volatile compounds in wine. A total of 138 compounds 
TABLE 3: Number of compounds extracted by fiber and variety of wine.

\begin{tabular}{lccccc}
\hline & PA & PDMS/100 & PDMS/DVB & CW/DVB & CAR/PDMS \\
\hline Gual & 124 & 116 & 126 & 129 & 116 \\
Malvasía & 128 & 122 & 124 & 130 & 121 \\
Listan blanco & 118 & 118 & 125 & 123 & 113 \\
Marmajuelo & 127 & 114 & 124 & 126 & 110 \\
\hline
\end{tabular}

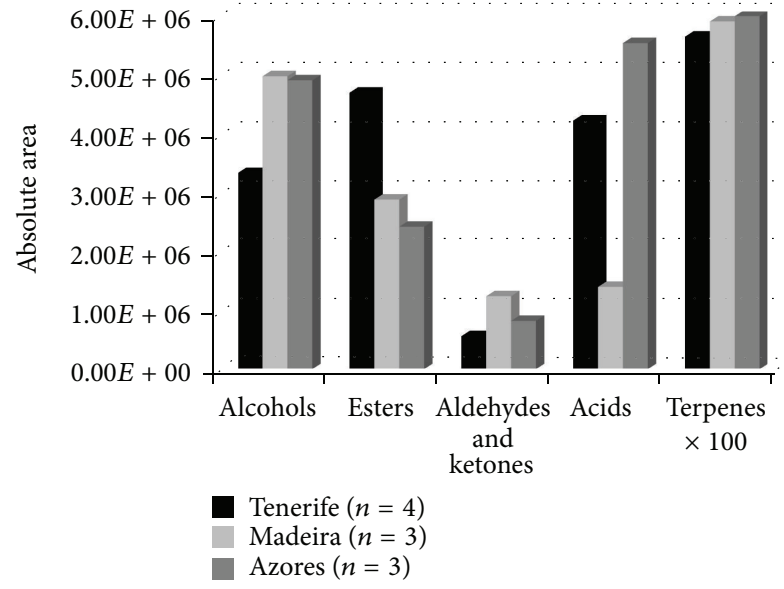

FIgURE 3: Absolute areas for different compound families in Verdelho wines.

were identified from different chemical families. The largest number of extracted compounds corresponds to alcohols and esters. Among the identified compounds, there are a number of important sensory compounds. All wines studied showed a content of $\beta$-damascenone, and whiskey lactone was detected too, unworthy of a young white wine without contact with wood, which could be a peculiarity of the wines from the Canary Islands. Malvasía variety presents the larger number of compounds detected. A comparative study between Tenerife, Madeira, and Pico Islands showed differences in compounds content mainly in alcohols, esters, and acids.

\section{Acknowledgments}

This work has been funded by the Spanish Comisión Interministerial de Ciencia y Tecnología (CICYT), project AGL 2003-04911/ALI. The authors acknowledge Bodegas Viñatigo (Tenerife) for sample supply.

\section{References}

[1] B. Plutowska and W. Wardencki, "Application of gas chromatography-olfactometry (GC-O) in analysis and quality assessment of alcoholic beverages-a review," Food Chemistry, vol. 107, no. 1, pp. 449-463, 2008.

[2] X. Yang and T. Peppard, "Solid-phase microextraction for flavor analysis," Journal of Agricultural and Food Chemistry, vol. 42, no. 9, pp. 1925-1930, 1994.
[3] M. E. Miller and J. D. Stuart, "Comparison of gas-sampled and SPME-sampled static headspace for the determination of volatile flavor components," Analytical Chemistry, vol. 71, no. 1, pp. 23-27, 1999.

[4] T. Hyötyläinen and M.-L. Riekkola, "Sorbent- and liquid-phase microextraction techniques and membrane-assisted extraction in combination with gas chromatographic analysis: a review," Analytica Chimica Acta, vol. 614, no. 1, pp. 27-37, 2008.

[5] B. Plutowska and W. Wardencki, "Aromagrams-aromatic profiles in the appreciation of food quality," Food Chemistry, vol. 101, no. 2, pp. 845-872, 2007.

[6] J. J. Rodríguez-Bencomo, J. E. Conde, M. A. RodríguezDelgado, F. García-Montelongo, and J. P. Pérez-Trujillo, “Determination of esters in dry and sweet white wines by headspace solid-phase microextraction and gas chromatography," Journal of Chromatography A, vol. 963, no. 1-2, pp. 213-223, 2002.

[7] J. J. Rodríguez-Bencomo, J. E. Conde, F. García-Montelongo, and J. P. Pérez-Trujillo, "Determination of major compounds in sweet wines by headspace solid-phase microextraction and gas chromatography," Journal of Chromatography A, vol. 991, no. 1, pp. 13-22, 2003.

[8] M. Mestres, M. P. Martí, O. Busto, and J. Guasch, "Simultaneous analysis of thiols, sulphides and disulphides in wine aroma by headspace solid-phase microextraction-gas chromatography," Journal of Chromatography A, vol. 849, no. 1, pp. 293-297, 1999.

[9] M. Mestres, M. P. Martí, O. Busto, and J. Guasch, "Analysis of low-volatility organic sulphur compounds in wines by solidphase microextraction and gas chromatography," Journal of Chromatography A, vol. 881, no. 1-2, pp. 583-590, 2000.

[10] J. S. Câmara, M. Arminda Alves, and J. C. Marques, "Development of headspace solid-phase microextractiongas chromatography-mass spectrometry methodology for analysis of terpenoids in Madeira wines," Analytica Chimica Acta, vol. 555, no. 2, pp. 191-200, 2006.

[11] R. Noguerol-Pato, C. González-Barreiro, B. Cancho-Grande, and J. Simal-Gándara, "Quantitative determination and characterisation of the main odourants of Mencía monovarietal red wines," Food Chemistry, vol. 117, no. 3, pp. 473-484, 2009.

[12] Z. Zeng, H. Zhang, T. Zhang, S. Tamogami, and J. Y. Chen, "Analysis of flavor volatiles of glutinous rice during cooking by combined gas chromatography-mass spectrometry with modified headspace solid-phase microextraction method," Journal of Food Composition and Analysis, vol. 22, no. 4, pp. 347-353, 2009.

[13] P. Alanis, S. Ashkan, C. Krauter, S. Campbell, and A. S. Hasson, "Emissions of volatile fatty acids from feed at dairy facilities," Atmospheric Environment, vol. 44, no. 39, pp. 5084-5092, 2010.

[14] M. Bueno, L. Culleré, J. Cacho, and V. Ferreira, "Chemical and sensory characterization of oxidative behavior in different wines," Food Research International, vol. 43, no. 5, pp. 1423-1428, 2010.

[15] L. Armada, E. Fernández, and E. Falqué, "Influence of several enzymatic treatments on aromatic composition of white wines," 
LWT-Food Science and Technology, vol. 43, no. 10, pp. 15171525, 2010.

[16] S. J. Pérez Olivero and J. P. Pérez Trujillo, "A new method for the determination of carbonyl compounds in wines by headspace solid-phase microextraction coupled to gas chromatographyion trap mass spectrometry," Journal of Agricultural and Food Chemistry, vol. 58, no. 24, pp. 12976-12985, 2010.

[17] V. Ferreira, N. Ortín, A. Escudero, R. López, and J. Cacho, "Chemical characterization of the aroma of Grenache rosé wines: aroma extract dilution analysis, quantitative determination, and sensory reconstitution studies," Journal of Agricultural and Food Chemistry, vol. 50, no. 14, pp. 4048-4054, 2002.

[18] R. López, N. Ortín, J. P. Pérez-Trujillo, J. Cacho, and V. Ferreira, "Impact odorants of different young white wines from the Canary Islands," Journal of Agricultural and Food Chemistry, vol. 51, no. 11, pp. 3419-3425, 2003.

[19] K. B. Shure and T. E. Acree, "Changes in the odor-active compounds in Vitis labruscana cv. Concord during growth and development," Journal of Agricultural and Food Chemistry, vol. 42, no. 2, pp. 350-353, 1994.

[20] J. Marais, C. J. van Wyk, and A. Rapp, "Effect of sunlight and shade on norisoprenoid levels in maturing weisser Riesling and Chenin blanc grapes and weisser Riesling wines," South African Journal for Enology and Viticulture, vol. 13, pp. 23-32, 1992. 

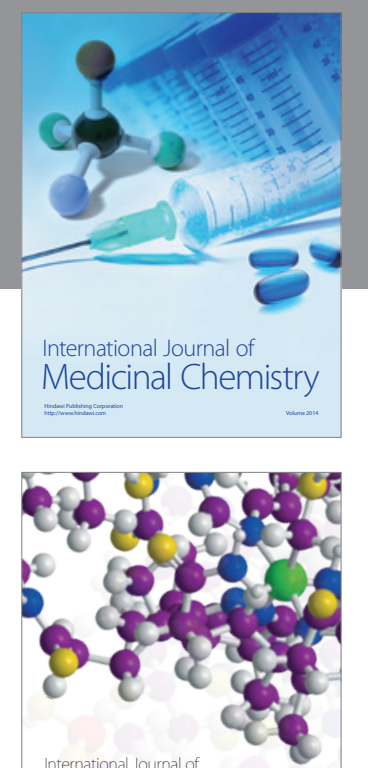

\section{Carbohydrate} Chemistry

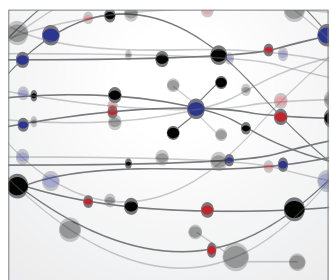

The Scientific World Journal
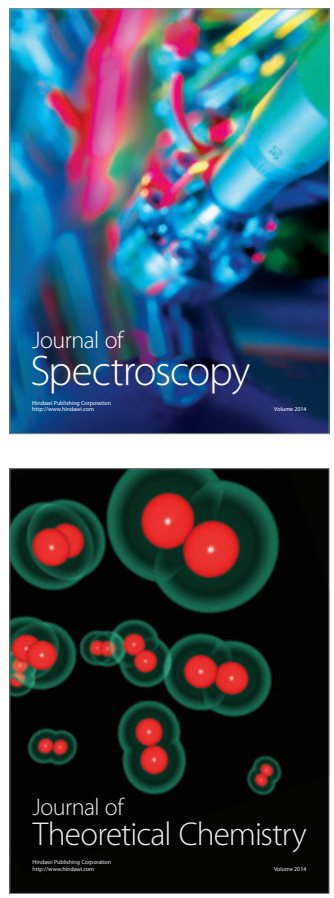
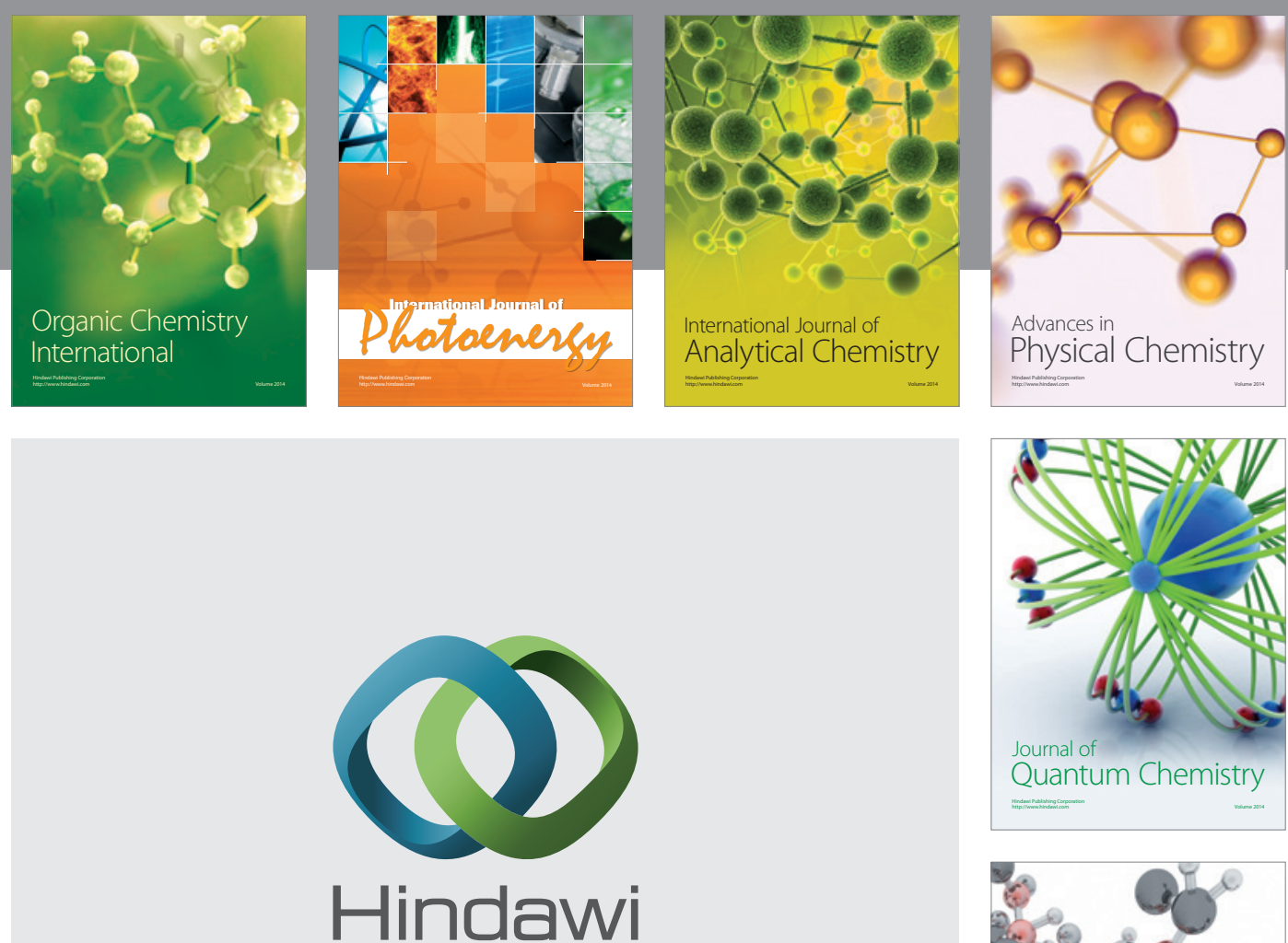

Submit your manuscripts at

http://www.hindawi.com

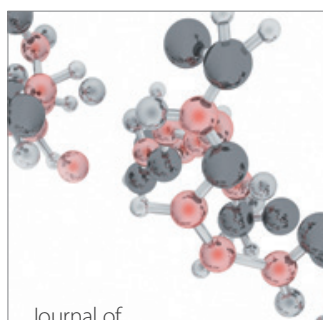

Analytical Methods

in Chemistry

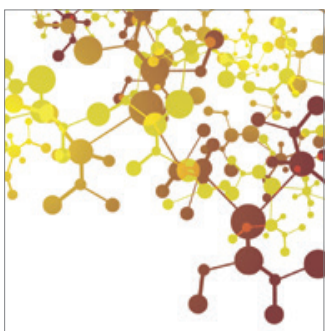

Journal of

Applied Chemistry

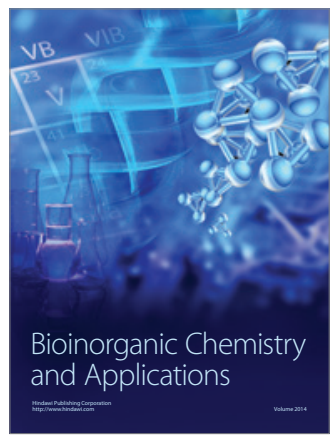

Inorganic Chemistry
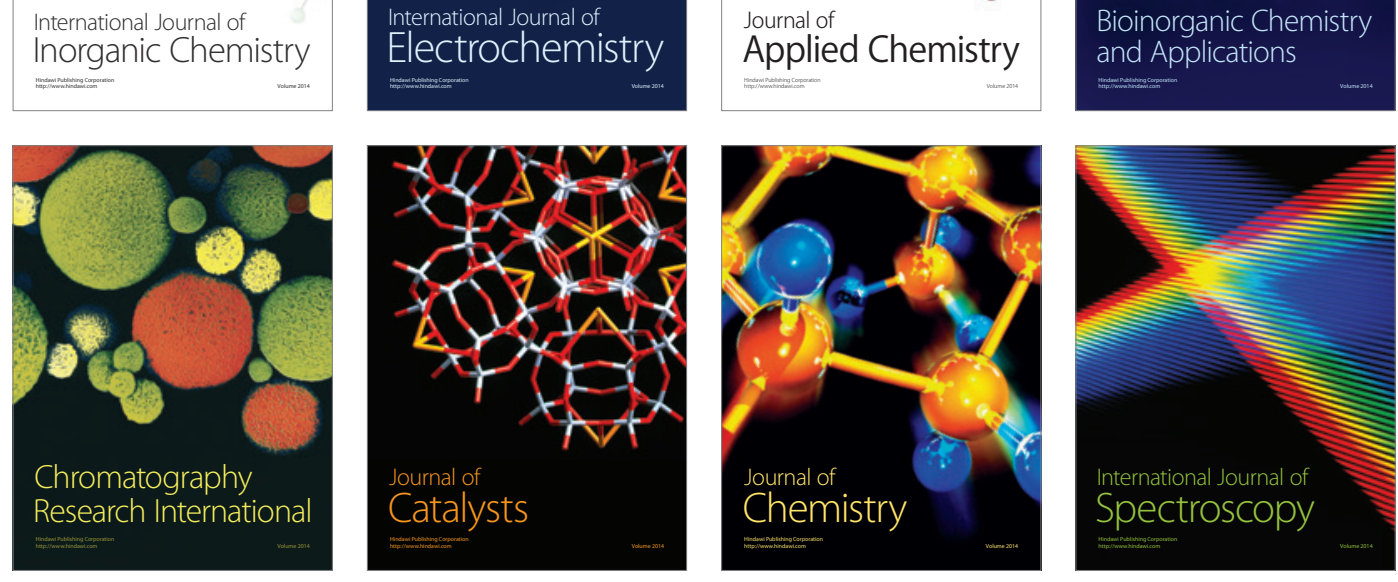